\title{
Making quantized images appear smooth: Tricks of the trade in vision research
}

\author{
ROBERT L. SAVOY \\ Rowland Institute for Science, Inc., Cambridge, Massachusetts
}

\begin{abstract}
Digital image-processing systems combined with color raster television technology are opening new worlds for the visual psychophysicist. Some of the inherent limitations and practical hazards of these technologies are explored. Techniques for circumventing some of the current limitations are presented, especially with regard to the issue of real-time variation of visual images.
\end{abstract}

This paper is divided into two parts. In the first part, I examine the question: To what extent can we generate arbitrary visual stimuli using current computer imageprocessing systems? This question can best be approached by making analogies between vision and audition. For both vision and audition, the problem is divided (somewhat idiosyncratically) into four issues: (1) abstract representation of the stimulus (e.g., an equation or algorithm); (2) explicit representation of the stimulus (e.g., pixel values); (3) communication of the explicit representation to a physical transducer; and (4) physical transduction. Limitations of current theory or hardware are pointed out for each issue.

In the second part of the paper, I describe several instances (from actual practice) in which various techniques were used to generate stimuli that were not what the underlying hardware devices were designed to generate; in short, I will document various tricks used to get around some of the limitations. Attention will be focused on a specific image-processing computer system, but the ideas are of wide applicability, given the current generation of image processors. At some point in the future, there may be image processors and displays that are designed explicitly for use in psychophysical vision research; in the meantime, however, the experimenter can generate a surprisingly general collection of stimuli despite hardware constraints.

\section{The Dream of Psychophysical Omnipotence}

What is needed to be able to create, in a laboratory, any visual stimulus? What information rate is required? How can the information be stored, communicated, and transformed into light? In addressing these questions, it is helpful to first consider the analogous questions for audition, using data summarized by Green (1976).

The ideas presented in this paper developed out of the pleasurable and stimulating process of performing experiments with, among others, John McCann of Polaroid Corporation, Suzanne McKee of SmithKettlewell, Eugene Switkes of the University of California at Santa Cruz, Jonathan Frankle of the University of California at Berkeley, and Michael Burns and Edwin Land of the Rowland Institute. I thank the Rowland Institute for Science for its ongoing support.

Reprint requests should be sent to Robert L. Savoy, Rowland Institute for Science, 100 Cambridge Parkway, Cambridge, MA 02142.
An arbitrary auditory stimulus has two dimensions: intensity and time. For most purposes in psychophysics (i.e., ignoring such things as bone conduction), if we specify intensity as a function of time at two points in space (one for each ear), we have specified the auditory stimulus completely. Furthermore, the required bandwidth for an auditory stimulus is $20 \mathrm{kHz}$. If we were to specify an auditory stimulus at discrete points in time separated by, for example, $1 / 40,000 \mathrm{sec}$ (twice the maximum frequency), then any errors introduced by temporal quantization would be irrelevant, because the auditory system cannot respond to them. (This is not the whole story, as temporal differences in phase between the two ears can be detected at finer temporal resolution.) Audible sound pressures cover a range of $10 \mathrm{log}$ units, and intensity discrimination is on the order of $1 \%$. If we were to represent intensity using a 6-bit exponent and a 10-bit mantissa, any errors introduced by quantization of intensity would be inaudible. Thus, only 640,000 bits per second per ear are required to perfectly represent an arbitrary auditory stimulus. This rate is orders of magnitude below the speeds of modest computers.

Does this mean that digital computers make auditory omnipotence a reality? Not quite, but they come very close. Consider the issues itemized in the introduction:

1.Abstract representation. Abstract representation of sounds is the current focus of much work in audition. On one hand, it is easy (both mathematically and computationally) to describe a pure tone. Indeed, it is so easy to compute a pure tone that an explicit representation is sometimes not used at all; each point for the explicit representation is computed in real time during stimulus presentation. The same is true for various combinations of tones and types of noise. On the other hand, the abstract representation problem is harder for music and speech. Substantial progress is being made in synthesizing both from various abstract representations, but the state of the art remains recording live performance or speech.

2. Explicit representation. Digital technology in the form of memory (magnetic tape or compact disks) permits a perfect (explicit) representation of the stimulus. The noise and dynamic range problems of analog storage tech- 
niques (e.g., recording on magnetic tape and phonograph records) are problems of the past.

3. Communication. Digital technology permits perfect communication of the explicit, stored representation to the transduction device.

4. Transduction. Considerable effort has gone into building systems, both for psychophysics and for entertainment, that convert the electrical signal into sound. Suffice it to say, without going into detail, that no one system can perfectly reproduce $10 \mathrm{log}$ units of intensity; however, from the perspective of psychophysics, reproduction quality from headphones is generally excellent.

The reason for describing these issues in audition at such length is to set the stage for a contrasting description of these issues in vision. Although an auditory stimulus has only two dimensions, intensity and time (for each ear), a visual stimulus requires six dimensions (for each eye). As in audition, there is a temporal dimension in vision; however, there are three intensity dimensions in vision, compared with only one in audition. These intensity dimensions correspond to the three degrees of freedom of color (e.g., red, green, and blue television guns or the psychological attributes of hue, saturation, and lightness). Most striking, of course, is the fact that vision has two spatial dimensions that are essentially absent from the auditory stimulus.

What is the information rate required for an arbitrary visual stimulus? The temporal resolution is at least $60 \mathrm{~Hz}$. (In fact, this temporal resolution is sufficient for sequences of continuous tone images, but is adequate neither for a few psychophysical tasks, such as masking and fine motion discrimination experiments, e.g., McKee \& Welch, 1985, nor for neurophysiological work, e.g., by Rodieck, 1983.) The intensity range is roughly $10 \mathrm{log}$ units, and intensity discrimination is normally on the order of $1 \%$, but $.1 \%$ is attainable for optimal stimuli. Using the same numerical scheme as outlined for audition, 250,000 bits per second per point would specify a visual stimulus perfectly (except as noted above). How many points are required to account for the two dimensions of spatial resolution? That question is less easily answered, as visual resolution varies across the retina. The point-by-point resolution in the fovea is about $1 \mathrm{~min}$ of arc. If that resolution obtained over the whole visual field, it would correspond to more than $\mathbf{5 0}$ million points. Since there are only about 6 million cones and 1 million optic nerve fibers, it is unlikely that such resolution is needed. If the 6 million cones are taken as a rough upper bound to the spatial resolution needed, an arbitrary image would require $1.5 \times 10^{12}$ bits per second per eye. Even in terms of explicit representation, this number is well beyond current digital technology. On the other hand, this combined degree of spatial, temporal, and intensity resolution and range is rarely, if ever, needed in a single sequence of images, even by psychophysicists. In practice, most currently available digital systems use 8 bits of intensity resolution per color and 1 million pixels of spatial resolution per time frame, although there is one new system that supplies 12 bits per pixel (described by Robertson, 1986). A given frame can be presented at $60 \mathrm{~Hz}$, and 10-bit digital-to-analog converters (DACs) can increase the effective intensity resolution. This corresponds to roughly 1.8 billion bits per second. Such a quantity of information can be explicitly represented, communicated, and transformed to light with present technology; however, to actually present a completely different (i.e., independent) picture every $1 / 60 \mathrm{sec}$ would require vast amounts of memory. It is not possible, except on the fastest serial or newer parallel machines, to generate such detailed images at that information rate.

Does this mean that visual omnipotence is an impossible dream? Let us consider the four issues again.

1. Abstract representation. Abstract representation of vision is a problem of long-standing interest. On one hand, it is a straightforward issue in the context of traditional psychophysics. Targets for increment threshold, bipartite fields for colorimetry, sinusoidal gratings, Gabor functions, and so forth have simple and elegant abstract representations. On the other hand, abstract representations for an arbitrary visual image (a city street scene, a pastoral farm, a face, a ballet) are at the heart of one of the most difficult and active areas of research in artificial intelligence, philosophy, and cognition (e.g., Pinker, 1984). At present, such natural images are generated using analog techniques, such as cameras and film or videotape. Bandwidths of these technologies (at least in their current implementations) are well below the spatial, temporal, and intensity ranges described earlier for perfect representation of images.

2. Explicit representation. In principle, this problem has been solved. It is simply one of memory; however, the amount of memory required is staggering. Videotape or analog videodisk technology make it possible to store a continuous presentation of images for entertainment (e.g., normal commercial television rates corresponding to roughly 20 million bits per second). But the digital technology that can represent the higher spatial $(1,024 \times$ $1,024)$, temporal $(60 \mathrm{~Hz})$, and intensity $(8 \mathrm{bit} / \mathrm{color})$ resolution that psychophysicists require and that current CRT displays can handle requires 1.5 billion bits per second. The much-heralded 1-gigabyte videodisks that will soon be available to store digital data will hold about $6 \mathrm{sec}$ worth of such images. Given these numbers, very highresolution movies are not likely to be stored digitally.

3. Communication. Communication, too, is not a problem in principle, but for digital systems it can be a problem in practice. Hardware is available that can transfer information at the necessary rates, but it is rarely available for communicating from conventional magnetic disk storage or memory, via the CPU, directly to the transducer. Instead, the information goes from the CPU to the image processor prior to encoding in analog television form. The communication link from CPU to image processor is often quite slow, with consequences that will be examined in detail in the second part of this paper. 
4. Transduction. Transduction of signals into images is the most substantial obstacle to the dream of psychophysical omnipotence. Cathode ray tubes, particularly shadow-mask color tubes, are the principal form of transduction for digital images, and there are substantial limitations imposed on the spatial resolution, spatial extent, spatial uniformity, intensity range, chromaticity range, and temporal control of such displays, as I will explain below. Although all of the CRT limitations are real, some are both less intrusive and more amenable to ongoing technical improvement than are others. For instance, spatial resolution in the digital images used to generate the signals sent to color shadow-mask CRTs has gone from the $512 \times 512$ pixels of 10 years ago to the commercially available $1,024 \times 1,024$ pixels of today, and experimental systems exist that have up to $2,048 \times 2,048$ pixels. (The shadowmask itself introduces quantization in the spatial domain, but it is not normally limiting as it is finer than that used in the image representation.) Frame rates have gone from $30 \mathrm{~Hz}$ (interlaced at $60 \mathrm{~Hz}$ ) to noninterlaced $60 \mathrm{~Hz}$. Given the associated increase in spatial resolution, this increased temporal resolution is impressive. Improved spatial uniformity and digital and automatic control of convergence have accompanied these developments. The intensity range of a CRT is good, typically from $0-100 \mathrm{~cd} / \mathrm{m}^{2}$, which represents a reasonably bright object.

That is the good news about CRTs, but there is also bad news. First, although state-of-the-art CRTs have good spatial uniformity and well-controlled power supplies, some older CRTs are astonishingly bad. Indeed, some even have automatic controls built in that make vision work impossible. For instance, some monitors include circuitry that averages the intensity of an entire display and automatically adjusts intensity to make that average fall into some range. Obviously, such circuitry must be disabled before trying to calibrate the CRT or use it for experiments. Some displays have circuitry that controls average contrast. Independence of the color guns must also be examined during calibration. Two things lead to interaction between color guns. One is imperfect alignment with the shadow-mask, so that the red gun, for instance, sends electrons to the green and blue phosphors. Another is the electrical power drain on the supply to one gun when another is changed. Finally, the center of the tube face is likely to be two to four times brighter than the sides or corners in many CRTs. This spatial nonuniformity is normally gradual and, therefore, rarely causes problems when working with continuous tone images.

Additional bad news is based on the highly nonlinear input-voltage versus output-light-intensity characteristics of CRTs. The nonlinearity can be compensated for, but the price is typically a loss of intensity resolution. This issue will be examined later in some detail.

Finally, there are several attributes of CRTs that are more or less inherent in the basic design and lead to limitations that are not likely to be changed. The spectral composition of any stimulus generated by a CRT is a linear combination of the three phosphors' spectra. This limits the chromaticity coordinates to a triangle bounded by the coordinates of the phosphors. Although this range is substantial, it lies well within the boundaries determined by monochromatic light. Such a limitation is negligible when attempts are made to reproduce continuous-tone natural scenes, but it makes a wide range of classical psychophysical experiments impossible to perform.

Another negative attribute is the spatial extent of the displays. One would like to be able to control the entire visual field of a subject. Obviously, today's CRTs cannot do that. Designing a tube large enough and curved enough (in the opposite direction to which CRTs are normally curved) to encircle the front of a subject's head would place great demands on the glass tube itself as well as on the electronics that control the path of the electrons within the tube. Yet there is considerable demand for some spatially extended display, especially for flight simulators (Haber, 1986). One approach is to use multiple CRTs. Another approach is to use optical color projection systems, based on three-monochrome CRTs. The potential advantages of such systems include increased size, greater spatial resolution, and more flexible spectral control (due to the ability to interpose color filters between the monochrome CRTs and the optics used for projection). Difficulties include brightness, spatial uniformity, and registration of projection systems, although improvements are rapidly being made in all these areas.

One of the most important problems with shadow-mask CRTs is the use of rastering. In return for the ability to present complicated spatial images, this technique places substantial restrictions on more general sorts of temporal control. (Rodieck, 1983, describes this problem in detail in the context of neurophysiological work. He ultimately chose to modify the color CRT to produce frame rates faster than $60 \mathrm{~Hz}$ by trading off spatial extent.) As a result, virtually all work on the psychophysics of motion and masking, when CRTs are used at all, is performed using monochrome CRTs that are not driven in a raster mode. There are no reasons, in principle, why color shadowmask CRTs could not be driven in a vector mode, but the cost of developing the relevant electronics would be prohibitive. (One kind of color CRT that does work in vector mode is a beam-penetration, rather than shadowmask, system; the energy of the electron beam is used to control color by penetrating to layers of different phosphors. This system has been used almost exclusively in flight simulators, where even minimal color shading is very helpful and fast temporal control is essential. Its color limitations rule it out for most vision work.)

Many of the technical issues raised briefly above were examined in detail at a recent symposium devoted largely to the issue of color CRTs and vision research. A special issue of Color Research and Applications (1986) reports the papers presented at this meeting. The paper by MacLeod (1986), in particular, lists some other problems inherent to CRTs. Nonetheless, the overall conclusion seems to be that color CRTs, despite their limitations, are finding wider and wider use, including clinical applica- 
tions (e.g., Cavanagh, Maurer, Lewis, MacLeod, \& Mather, 1986).

Where does this leave the psychophysicist who dreams of omnipotence with respect to visual stimuli? It says that one set of hardware will not suffice. To generate intricate, mathematically defined images quickly and easily, one needs a digital image-processing system and CRT display. But if the investigator also wishes to perform traditional experiments using the full range of visual intensities, monochromatic light, fine temporal control, and very large visual fields, a separate set (indeed, probably several sets) of hardware is needed. Finally, to use digital systems to generate nonstatic images, the researcher normally has to sacrifice something-intensity resolution, temporal resolution, and/or spatial resolution.

\section{CASE STUDIES}

Having described some of the general limitations of digital image processing as it is related to visual psychophysics, I will spend the remainder of the paper examining case studies from my own work in which these limitations are circumvented. Most, if not all, of these techniques are not novel. Indeed, to someone accustomed to doing vision work with image processors of the sort described below, the techniques may seem familiar and perhaps even elementary. On the other hand, my impression is that it takes considerable practice before such techniques suddenly seem obvious. The present description is intended to facilitate that transformation.

The principal component of the particular technology I have been using is a Model 70 image processor manufactured by International Imaging Systems. It has, in terms of memory, $512 \times 512$ spatial resolution, and each pixel of the $512 \times 512$ image is represented by eight bits of intensity information. The chassis and associated hardware of the system can accommodate the memory for as many as 12 independent images, although the systems I have used included only 3 or 5 such image memories. A trackball and digitizing tablet are used for interactive input. In addition to writing directly to image memory from a host computer, the Model 70 has a digitizer for converting TV signals from a television camera to image memory. A PDP-11/60 or VAX-11/780 (both from Digital Equipment Corporation) have served as host computers.

The architecture of the image processor includes several other features that are fairly standard. One is horizontal and vertical scrolling-the ability to shift the picture by an arbitrary number of pixels, with the portion of the picture thus removed from the screen reappearing on the other side (usually called wrap-around). Another feature is zooming via pixel replication-multiplying the linear dimensions of each pixel by 2,4 , or 8 so that one part of the image resultantly occupies the whole display. In both instances, the actual values in image memory remain unchanged. Only the algorithm that transforms those pixels to an analog television signal changes. For scrolling, the horizontal and vertical location of the pixel where reading begins is varied. For zooming, a given pixel is read multiple times.

By far the most important feature of the architecture is the use of look-up tables. Let me engage in a brief digression to remind the reader of the function of lookup tables. (See, e.g., Rodieck, 1983, for a more extended discussion.) During the presentation of each frame of the image (as happens every $1 / 30 \mathrm{sec}$ on my system), each pixel in (each) image memory is read and used to generate part of the analog television signal. In the course of this calculation, one or more transformation tables can be applied to the raw number in memory. Thus, for example, if the number in memory were 123 , and the 123 rd element of the table were 241 , then the value ultimately sent to the DAC would be 241 . This transformation would be applied to all memory locations that contained the value 123. Although it takes considerable time to change all the memory locations in an image with a quarter of a million pixels, it only takes a tiny fraction of that time to change the 256 entries in a look-up table. The transformations that this architecture can implement are limited, but still very useful, as will be indicated shortly. For now, let me note that look-up tables are routinely used to compensate for the drastically nonlinear intensity versus voltage characteristics of CRTs, a procedure that is usually referred to as gamma correction.

The image processor that I used not only has three lookup tables (one per color) prior to the digital-to-analog conversion stage in the generation of color television signals, but it also has an additional three tables for each image memory (i.e., 15 tables in all for my system with five image memories). Thus, each image could send information, transformed by its own table, to each color gun independently, where the information from the various images is summed prior to the gamma-correction look-up table. This architecture permits considerable real-time flexibility. (The image processor has several other features that are less standard and not relevant to the present discussion.)

One characteristic of the architecture is particularly important in almost all of the examples that follow. It is the problem of communication between the host and the image processor during real-time presentation of stimuli. Recall that each frame is being displayed on the CRT every $1 / 30 \mathrm{sec}$. In fact, this system uses an interlaced scheme, presenting the frame as two fields, each of which consists of every other line in the frame. Thus, the fields are presented every $1 / 60 \mathrm{sec}$. During the time that the analog television signals are being sent, it is impossible to send information to the processor without distorting the image on the CRT. (This is, essentially, a bug in the hardware design that may have been fixed in subsequent models.) But even if there were no distortion during transmission, $1 / 60$ of a second later a new frame is going to be displayed and all the information relevant to that new frame must have been transmitted before generation of the analog television signal commences. In short, there are at most $16 \mathrm{msec}$ to compute and transfer all the in- 
formation that will change the appearance of an image from one frame (or field) to the next. That is not enough time to write new information in many pixels.

On our system, the situation is even worse. Because of the problem cited above, even the $16 \mathrm{msec}$ are not available. Only 2-3 msec, during which the television signal is completely blanked (during vertical retrace, prior to each raster), are available for transmission of data to the processor from the host computer. (Fortunately, and critically, the hardware does inform the device driver in the host when the screen is actually blanked so synchronization is possible.) Limitations of the host and I/O interface to the image processor result in the ability to send less than 200 16-bit words per frame, using the VAX as host. On an earlier system with the PDP-11/60 as host, that number was just over 300 , which allowed us to send a full 256-element look-up table during the blank period.

The reader should keep in mind that some of the details of the technology are changing rapidly. Indeed, International Imaging Systems has not produced the Model 70 for a number of years now, replacing it with more advanced processors having greater spatial and intensity resolution. But the primary intended use-analysis of static LANDSAT and medical images-and the architecture designed for such use have not changed drastically. The basic utility of look-up tables, scrolling, and pixel-zoom, and the basic constraints of digital image processing, communication, and color CRTs, seem to be relatively stable. It is useful to see how to use the former to overcome the limitations of the latter.

\section{Case 1: Changing Color Interactively While Maintaining Luminance}

The first example is a simple color-cancellation technique used to measure the strength of a contingent color aftereffect (Savoy, 1984). The stimulus was a spatial structure consisting of a checkerboard-like pattern, where each "check" was actually a square wave grating oriented at plus or minus $45^{\circ}$, and the whole pattern was surrounded by a white annulus. The color of the light bars in each square wave was being changed interactively. All the light bars of a given orientation were changed the same way. Although this pattern was relatively simple, and only two sorts of areas were changing color, those areas contained more than 20,000 pixels. Changing the red, green, and blue separation values for such a stimulus would require orders of magnitude more speed than I had available. Instead, a single image memory was used with only a few values. The diagonal $+45^{\circ}$ stripes had one value (e.g., 1), the diagonal $-45^{\circ}$ stripes had another value (e.g., 2), and the surrounding annulus, the background, and the dark stripes each had one value. When the subject moved a trackball, the changing horizontal value (vertical values of the trackball were ignored) was used to calculate the desired change in color of the two areas. For instance, trackball motion caused green light to be added to one line orientation and an equal amount to be subtracted from the other orientation. The amount of red and blue light was simultaneously adjusted for each orientation so that the calculated CIE luminance of both test areas remained constant and equal to that of the fixed achromatic annulus. Thus, subjects changed saturation along one hue dimension (magenta/green) without altering the luminance of either test area. With the hardware used, each trackball change had to be sampled; then some moderately elaborate chromaticity and luminance calculations were performed, and the appropriate values were written in the 1 and 2 locations of the red, green, and blue look-up tables. (In fact, only the three look-up tables for the color guns were used, since the gamma correction could be incorporated in the calculation described above.) To do the calculation, $16 \mathrm{msec}$ sufficed, and 2-3 msec were easily sufficient to send the 616 -bit words that indicated new values for the look-up tables. Actually, an additional 8 words per table were used by the image processor to keep track of which of its many tables, registers, and memories the new data was intended to reach. Nevertheless, the total was only 30 16-bit words.

The basic theme here-modifying the appearance of an image in real time by changing a table, rather than by changing the image memory itself-is pervasive when image processors are used for psychophysical experiments. This example also highlights the additional freedom the experimenter has to transform a single parameter from the subject (such as the trackball value) to control a reasonably elaborate scheme for selecting the next stimulus. In this case, the scheme restricted chromaticity and luminance variation, but it could, in other contexts, be used to control staircases and so forth.

\section{Case 2: Smooth Changes in Space and Time}

As part of my thesis work (Savoy, 1980), I wanted to compare contrast thresholds obtained using a forcedchoice technique with thresholds obtained using a method of adjustment. Such work had been performed before (Kelly \& Savoie, 1973), and my work essentially replicated those results. In the present paper, however, I am not concerned with the psychophysical data itself (which indicated that the forced-choice technique gave slightly lower thresholds for vastly greater effort, while not changing the relative shapes of any curves), but rather with the technical problems that arose in using computer-generated raster images to do the vision experiments.

I was primarily interested in the spatial, rather than temporal, properties of the visual system and, therefore, wanted to minimize the influence of temporal transients. Furthermore, I wanted to minimize the influence of spatial transients not on the same scale as whatever spatial scale I was studying. The result was a desire to make the stimuli as smooth as possible in both time and space.

The working definition of smooth for these stimuli was Gaussian. The goal, stated mathematically, was to start with an infinite, one-dimensional sinusoidal grating that had to be presented on a finite-sized CRT for a finite period of time, and to make the transition from infinite 
to finite in a Gaussian manner. This required varying the contrast in both space and time.

This goal requires consideration of all three ways in which rasterized, digital images are quantized-in space, time, and intensity. This sort of quantization is amenable to formal mathematical analysis (e.g., Cowan, 1983) and to minimization of impact based on psychophysical grounds (e.g., Mulligan, 1986). In the present context, problems can arise in the generation of high-frequency stimuli because putative sine waves become triangular or square waves at high enough spatial frequency. Moreover, when the frequency is close to that defined by the pixel resolution, beats will be generated when frequencies correspond to a nonintegral number of pixels per cycle. At the other end of the spatial frequency scale, problems can arise in the generation of very low-contrast stimuli because sine waves become visibly square waves or staircase functions. This latter problem is essentially a consequence of the fact that human increment threshold for edges is rather good (close to .1\%). Approaches to this problem will be examined in Case 3. For the smoothed functions I was using, thresholds were never less than $1 \%$, so this problem was not serious.

Let me now describe the details of the stimulus generation. The stimuli consisted of sine wave gratings of various frequencies $f$ and contrasts $C$ presented in cosine phase at location $\left(x_{0}, y_{0}\right)$ gated by Gaussian filters centered at $\left(x_{0}, y_{0}\right)$ and of widths $W_{x}$ and $W_{y}$. Contrast was either controlled interactively (for the method of adjustment) or gated on and off smoothly (for the forced-choice procedure), where, as for the spatial dimension, smooth means Gaussian, centered at $t_{0}$ and of temporal width $W_{t}$. Although a mathematical sine wave has both positive and negative values, there is no such thing as a negative intensity of light. The actual stimuli were variations around some mean level $M_{0}$. Thus, the stimulus $S$ was:

$$
\begin{aligned}
S(x, y, t)= & M_{0}+ \\
& C e^{-\left[\left(t-t_{0}\right) / W_{t}\right]^{2}} \cdot e^{-\left[\left(y-y_{0}\right) / W_{y}\right]^{2}} e^{-\left[\left(x-x_{0}\right) / W_{x}\right]^{2}} \cos (2 \pi f x) .
\end{aligned}
$$

How is digital approximation to such an entity created? First, in order to think about a stimulus representation that is linear with respect to the above equation, we eliminate the nonlinearity of the CRT via the gammacorrection look-up tables. Next, we choose some number to correspond to the mean level $M_{0}$. Normally, a number near the middle of the range of available memory values (0-255) is used. It is usually a good idea to arrange for the mean and extreme values to fall equally spaced from each other and on exact image memory values, despite the fact that a small amount of image intensity resolution is wasted. Thus, the value 127 was chosen for the mean, and 0 and 254 were the extremes. The maximum contrast in this case would be $C=127$ and would correspond to a Michaelson contrast of 1 . (A fringe benefit is that the value 255 is available for other uses, such as making a background of arbitrary color.) Note that a higher or lower value for the mean could be used, but this would impose some limitations on the values of $C$ or on the effective contrast. If $M_{0}$ were greater than 127 , then the maximum obtainable Michaelson contrast would be less than 1 . If $M_{0}$ were less than 127, care must be exercised to be certain that $C \leq M_{0}$, or negative luminances would be requested. Also, although contrasts as high as 1 could be nominally set, the intensity quantization limitations would be revealed at extreme contrasts because the jump to the first nonzero intensity step would be very great.

Using the conventions listed above, the equation is used to generate an image memory at the maximum nominal contrast $\left(M_{0}=127 ; C=127\right)$. The spatial position and frequencies were chosen so that there was an integral number of pixels per cycle and so that all peaks and zeros fell exactly on pixels.

Contrast is varied via look-up tables. Recall that prior to reaching the gamma-correction look-up tables, there is another set of tables that can be used to transform the values in memory. Normally these tables are set to be a linear ramp of slope 1, that is, the identity function. By changing the slope of the tables, but still passing through the point $(127,127)$, the contrast of the stimulus is varied. In the method of adjustment, the user moved a trackball to indicate the desired contrast. There was enough time on our host to compute a new linear ramp for the look-up table in real time, but had there not been, the various linear ramps could have been precomputed and stored in the computer's memory. Then the trackball would have been used as an index to the appropriate ramp.

The smooth variation in time is performed using the same basic ideas, except that a sequence of contrasts is precomputed and shown for a variable amount of time. The contrasts and lengths of time (i.e., number of frames) are chosen so that the contrast-versus-time function is a quantized approximation to a Gaussian.

The preceding techniques apply to varying contrast for any image. I subsequently used it in studies on neural afterimages from natural scenes digitized using a television camera and the image processor.

The point of this example is that complicated functions of space and time can be smoothly varied in real time using look-up tables.

\section{Case 3: Increment Threshold and Contrast Sensitivity}

In both of the first two studies, for slightly different reasons, the quantization noise in intensity was not a problem. In the color-cancellation study, the intensity resolution was high enough that discrete jumps could not be seen. In the contrast threshold experiment, the smoothed functions were sufficiently difficult to see that the required contrast was fairly high.

On the other hand, when raw sine wave gratings near the optimal spatial frequency of the visual system are used, or when a simple edge is used, intensity resolution becomes a significant problem. Despite the fact that the system under consideration has a 10 -bit DAC for the final 
generation of the video signal, intensity changes are too large. Specifically, if we change the input to the DAC by a single unit across an extended edge (say from 700 to 701 ), the change is visible.

There are several ways to handle this problem. The best way is at the design stage of the image processor itself. It is easy to include an amplifier that allows the user to select which region of the $0-1 \mathrm{~V}$ range used in the television signal to map from the 10-bit DAC; that is, instead of having the DAC map the $[0,1023]$ input values to $[0,1]$ output volts, map them to, say, $[.5, .6]$ volts. In practice, of course, such a feature is only useful for vision research and, therefore, does not yet appear in actual devices.

This same sort of idea can be applied directly to the analog television signal after it leaves the image processor on the way to the CRT, but doing so is much more difficult. The television signal is composed of many parts, only one of which is the analog intensity component. There is also a host of timing and synchronization information that has nothing to do with the particular stimulus, but rather with rasterizing. Thus, the electronics cannot consist of only a simple amplifier. More electronics are needed to select the right part of the signal to transform. Then the transformation is simple, although fairly fast amplifiers are still needed. This is the general method for solving intensity resolution problems with CRTs. It has been implemented successfully in at least one laboratory (E. Switkes, personal communication, May, 1983).

Finally, there is the nonelectronic solution to the problem. Basically, a uniform field of light is added to the CRT display. This can be accomplished by putting a semisilvered mirror in front of the CRT display, tilted at $45^{\circ}$ from the plane of the CRT. A source of spatially uniform light is then mounted at $90^{\circ}$ from the plane of the CRT, facing the mirror. Since the intensity of this uniform field is added to that from the CRT, the contrast increment represented by each unit change in the DAC output is reduced. (One implementation of this idea, albeit with some slight differences, is illustrated in Figure 3 of Savoy \& McCann, 1975.)

The lesson from Case 3 is that working near the threshold of perception is difficult with these systems, because they are near their own performance thresholds. The trick is to add light or to modify the voltage range of the DAC outputs so that the hardware is not working near its intensity resolution limits.

\section{Case 4: Experiments in Color Constancy}

The following discussion is centered on a procedure called the "Color Mondrian Demonstration" (CMD) (after experiments by Land, 1974, and McCann, McKee, \& Taylor, 1976), because it makes use of stimuli that are somewhat reminiscent of the paintings of Piet Mondrian. Rather than temporal constraints, the central issues for Case 4 are calibration and limitations on the intensity range of the CRT.

In the context of projectors and reflectance targets, the CMD consists of the following procedure. Turn on the long (red), middle (green), and short wave (blue) projec- tors to illuminate a collection of rectangular papers called the "Mondrian." Measure the light being reflected from, for example, a gray paper and a red paper. Then adjust the intensity of the three projectors so that the light coming from the red paper in the new illumination is identical to the light coming from the gray paper under the previous illumination. Color constancy refers to the fact that the papers in the display (and the red paper, in particular), although not identical to their previous appearance, are nonetheless labeled with the same color name by observers. McCann et al. (1976) quantified this experiment.

The question is, how can we simulate these experiments using image processors and CRTs?

Obviously, there are some general issues related to calibration. One must know the energy-out versus voltagein for each color gun independently. Recalling a warning mentioned in the first part of this paper, one must be sure that the monitor does not automatically include gain or contrast control. This would be particularly disastrous in simulating CMD, as the gain controls would generally be the opposite of what the experimenter was trying to implement as a simulated change in illumination. But beyond these problems, there are several more subtle ones.

It is easiest to conceive of the digital version of the Mondrian as being composed of many areas, each with three hypothetical "reflectance" values, one value for each of the television phosphors. The three hypothetical "illuminators" have the known spectral characteristics of the three television phosphors, and variable intensity. The areas' "reflectances" are chosen so that the appropriate product of reflectance and intensity will yield a combined excitation of the three phosphors so that the tristimulus values are identical to those of the original paper-andprojector stimulus. Then we can, in theory, follow the analogous procedure for performing a CMD experiment. Two areas are selected, and the intensity of the "illuminators" is adjusted so that the tristimulus values of the second area in the new illumination are identical to those of the first area in the original "illumination." (Note that I have kept "reflectance" and "illumination" in quotes because these are merely convenient fictions for the purpose of calculation-no real object could reflect, for instance, $100 \%$ of the red television phosphor and $1 \%$ of the green phosphor, although these hypothetical reflectances could be chosen to correspond to such a situation.)

When we try to apply the preceding algorithm, certain problems arise. At one extreme, not enough energy may be available in a given television gun. That is, in changing the hypothetical illumination to make the second area have the specific tristimulus values that some other area had (something we can certainly do), we may require still other areas to have tristimulus values that are beyond the range of the television to produce. At the other extreme, an area may become so dark that the quantization of intensities becomes apparent.

The above procedure started with a collection of papers and illuminators that were broadband. (If narrow band illumination were used, there is a good chance that the 
resulting tristimulus values could not be generated via the relatively broadband CRT phosphors.) Having started there, however, the temptation is to generalize the situation on the television using areas that have more extreme chromaticities. In particular, using areas with hypothetical reflectances that are extreme (such as $100 \%$ in the long wave, but $1 \%$ in the middle wave) drastically exacerbates the problems presented in the previous paragraph. Such reflectance spectra may be useful in studying visual processes, although they are rarely found in natural objects.

One way to avoid this problem is to define the reflectances of the hypothetical areas so that no hypothetical "reflectance" is lower than, for instance, $10 \%$. Another way is to measure real objects for defining the reflectances. In either case, it will be necessary to make a judicious choice of the peak intensity of any area in the scene in its original illumination. The peak should be well below the maximum that the television can produce, so that when the simulated illumination is changed, increases in output can be generated.

An alternative, when the two reflectance areas are known in advance, is to choose the initial illumination in such a way as to guarantee that the necessary changes will be within the television range. (The idea is to decrease the original illumination for the gun whose output will have to be increased in the second illumination.) This algorithm is slightly different from the algorithm used in the projectors-and-papers version of the CMD. When using projectors, the illumination of one point is taken as given-its relative gain is set to $(1,1 ., 1$.$) . The gains$ for the other point are scaled up or down as needed, within the limits of the projectors. Since the television is calibrated at its maximum usable brightness, and since we normally like to display images using that full range, it is usually difficult to scale energies up. So the following is done for each color gun independently. Suppose that the red energy of the first point $\left(R_{1}\right)$ is higher than that of the second point $\left(R_{2}\right)$. Then, Illumination Condition 1 will use $\left(R_{2} / R_{1}\right)$ as its red gain, and Illumination Condition 2 will use 1.0 as its red gain. Thus, point 1 will send $R_{1} \times\left(R_{2} / R_{1}\right)$, that is, $R_{2}$ units of red gun energy to the eye in Condition 1 and point 2 will send $R_{2} \times 1.0$, that is, $R_{2}$ units of red gun energy to the eye in Condition 2. Similar calculations are performed for the green and blue guns. Thus, the gains applied are always less than or equal to 1.0 .

There are several advantages to performing the CMD using CRTs. Digitally generated images allow the experimenter to simulate experiments that are very difficult to do with a real Mondrian display. For instance, one can instantly isolate a given area by surrounding it with a uniform field. (This is the television analog to looking through a black tube to isolate some point from its surroundings.) This is a dramatic way to demonstrate the effects of changing the simulated illumination. It is also a good way to see if there are spatial interactions within the television. This is done by combining the electronically generated mask with a physical mask. The physical mask must be very black, for instance a board covered with black velour, with a hole for the test area. By rapidly switching the digital stimulus from the original complex scene with the test area, to a uniform white, gray, or black mask, and viewing only the test area through the physical black mask, one can get some idea of the spatial interactions of the electronics of the television. In particular, the effects of the automatic gain control circuits mentioned earlier, if they exist, will be strikingly apparent.

The lesson from Case 4 is that careful color calibration of the CRT can be thwarted by internal electronics and limitations on the high and low end of the intensity range of CRT color guns. These limitations must be anticipated in the design of experiments in color constancy, but the payoff in flexibility can make the effort worthwhile.

\section{Case 5: Random and Structured Motion}

The last theme, which is illustrated by a small collection of studies, is that of generating apparent motion on inherently discrete color CRTs.

Motion is the attribute of visual stimuli that this sort of digital image processor is least well-equipped to handle. The underlying information structure of a collection of pixels does not lend itself to the sorts of transformations that motion causes, except in the very simple case of linear translation of the entire image, which can be achieved using the scrolling feature.

As an aside, let me comment that motion is handled more naturally in a separate class of computer systems used, for example, in Computer Aided Design (CAD) and some branches of artificial intelligence research. In those systems, the three-dimensional characterization of objects and the ability to easily manipulate that representation in space are the critical problem, not the perfect rendering of the visual image that might correspond to the object. Thus, instead of images based on a collection of points (with excellent intensity resolution and good spatial resolution), CAD systems typically represent objects as a collection of vectors with, at the extreme, superb spatial resolution and no intensity resolution, that is, as white lines on a monochrome CRT. Rotating or translating part of an object whose image is represented by points is much more computationally intensive than the same transformations applied to a vector representation. In one sense, the matrix that corresponds to rotation, translation, and scaling of objects plays an analogous role in vector displays to that played by look-up tables in pixel-oriented image processors-it allows rapid change of relevant features at little cost in terms of information transfer. Even without the sophisticated digital hardware used in abstract representation of three-dimensional objects for $\mathrm{CAD}$, the monochrome CRT's high spatial resolution and very rapid temporal response make it ideal for some studies of vernier acuity, stereopsis, and motion.

In the present type of image-processing hardware, the biggest trade-off between space and speed, between memory and how fast you can do things, is evident in mo- 
tion, as seen in the discussion of videodisk digital storage capacity. But how does this trade-off apply to the image processor I have been using? Despite the fact that the image processor was fundamentally designed to analyze individual still images, there are many ways to obtain motion. Each involves some sort of trade-off of the features for which the system was designed.

For instance, suppose that for an application, the investigator does not care about intensity resolution but wants to retain spatial resolution. Then, instead of conceiving of the image memory as $5512 \times 512 \times 1$ byte frames, we can think of it as $40512 \times 512 \times 1$ bit planes. We could compute each black/white image (e.g., stick drawings of chemicals) separately for the 40 planes and then show them in sequence (via changing look-up tables) to simulate a little strip of animated movie. If the motion were periodic, this might suffice completely. For instance, 40 snapshots of a rotating molecule are normally adequate to give the impression of smooth rotation.

To go one step further, suppose that some spatial resolution could also be sacrificed. Then, using the pixelreplication zoom and scroll features, we could conceive of memory as $160256 \times 256 \times 1$ bit planes, with the ensuing increase in motion resolution or in duration.

Note that each frame must be displayed using only two colors in this scheme, but that these colors need not be black and white. The colors are arbitrary, and can be manipulated in real time using the techniques described for Case 1. As an example, Savoy and Burns (in preparation) combined the color and motion techniques to make a variety of heterochromatic brightness, minimum motion, and minimum stereopsis isoluminant settings for dots on a rotating sphere.

Suppose, on the other hand, that we needed all the spatial and intensity resolution, but were willing to settle for somewhat jerky and periodic motion. In fact, there are several situations that require such motion. The quadrature grating technique of Anstis and Cavanagh (1983) and the motion paradox demonstration of Adelson and Bergen (1985) both require grating motion in $90^{\circ}$ jumps from frame to frame. Four $512 \times 512$ images are sufficient to simulate such motion. Again, the basic stimuli are written to image memory first, and only tables need be varied to switch between them.

The preceding techniques are all forms of animationthere are some number of fixed, independently generated images, and we step through them. If we do not care about spatial or intensity resolution, we can have many steps. If we need lots of spatial and intensity resolution, we can only have a small number of steps.

There are a few interesting intermediate cases. Suppose the image is not changing in an arbitrary way from frame to frame, but in a more structured way. Case 2 showed that some kinds of temporal change could be simulated using a single image of high spatial and intensity resolution. That applies to some kinds of motion, too. For instance, as previously mentioned, linear motion can be simulated using the scrolling feature of most image processors.

Suppose we want to do something more complicated, such as smoothly rotate an image in the plane of the CRT. There are no commercially available systems that allow us to do that directly. On the other hand, rutation can be simulated for restricted classes of images. If the stimulus is constant along a collection of curves that are rotations of each other around the center of rotation, then the stimulus can be "spun" using look-up tables. For example, the "curves" could simply be radii from the center; the stimulus would then be independent of radial distance and simply depend on angle of rotation around the origin. A slightly more interesting case would be creating spiral curves, the sort of stimuli that generate expanding or contracting motion aftereffects. In all of these situations, the idea is to create an image that has values $0,1,2, \ldots$, $N-1$ along the individual curves from the center, where $N$ is the number of intensity levels in memory (typically 256). (The "curve" will necessarily increase in width as we move radially outward, and that loss in spatial resolution is the price paid to be able to rotate the final image.) Then we can map any function we want in the appropriate table to generate the desired stimulus, that is, the look-up table contains the functional shape desired for intensity while the image memory is used to index rotational position. If we "rotate" the table (i.e., move each element up the same amount and wrap around elements that fall out the top of the table back down to the bottom), then the image will appear to rotate.

If the constraints imposed by the given hardware cannot be overcome, other technologies must be considered. For instance, if we need genuinely random sequences over long periods of time, there is no way that we can compute, communicate, or store them in the way image processors normally work. But we can add a video recorder that has the capacity for recording one frame at a time. We will, at least with current technology, be sacrificing spatial and intensity resolution, but we will be able to generate a complicated stimulus that can be played back later. Indeed, this is the technology that is used by those who need truly random stimuli for performing Weiner kernel analysis of single-cell response to twodimensional white noise (e.g., Citron \& Emerson, 1983).

In some situations involving random noise, however, the noise does not have to be random over long periods of time. For instance, there is a phenomenon that can be seen using the white noise that appears on a conventional television when it is tuned to a nontransmitting station. If a neutral density filter or a small aperture is placed in front of one eye, the viewer sees (for reasons that are related to, but not completely explained by, the Pulfrich effect) motion that seems to be drifting cylindrically around the center of the screen. Dots moving in the direction indicated by the eye that has the neutral density or aperture in front of it appear to be closer to the observer than does the screen, and dots moving in the opposite 
direction appear to be behind the screen. In studying this phenomenon, the researcher needs to have more control over the stimulus-it is not adequate to simply use the noise generated by tuning to a nontransmitting station.

We can successfully simulate such noise on the system under discussion, and there are a number of ways to do it. If we do not mind the dot density being much lower than the $50 \%$ used for actual television noise, we can create it with a single $512 \times 512 \times 8$ bit image by writing random numbers (in the range $[0,255]$ ) in the image and then turning on a random subset of the look-up table for that image at each time frame. (By turning on, I simply mean writing 255 in various entries of the table.) If we do not care that dot density varies from frame to frame, then we will generate something like $\mathbf{2 5 5}$ different images. More realistically, if we want density to stay fairly constant, we could turn on, for instance, random subsets of 10 elements from the table. That would still generate some $10^{23}$ different patterns. Of course, these would not be random patterns, because any two table settings that turned on the same value would be activating one subset of pixels that was identical. A more reasonable use of this scheme would be to settle for 256 different random patterns, one for each value of the table. The point, as usual, is that only the look-up table need be changed to alter the image.

If random dots with higher density are needed, we can use the 40-snapshots approach described earlier for simulating structured motion. Furthermore, if a factor of 2 can be sacrificed in linear spatial resolution, 160 snapshots could be used. Even presenting these stimuli for $1 / 30 \mathrm{sec}$ each, this constitutes about $5 \mathrm{sec}$ of genuinely random noise. Ten seconds of random noise are obtained if each frame is repeated so that it lasts $1 / 15 \mathrm{sec}$. Either way, it turns out that this is sufficient to generate the desired effect without introducing perceptually apparent artifacts. I am asserting neither that the subjects cannot tell that the stimuli are periodic, nor that the subjects cannot distinguish the stimuli from actual television noise, but simply that it does not matter with respect to the perceptual phenomena of interest.

If we have an image processor with single-pixel scrolling capabilities, there is a much more elegant solution to the problem of simulating random noise. (This algorithm is described for noise with 2 intensity levels, but is easily generalized to 16 levels using the given hardware.) We generate two independent random fields of $1 \mathrm{~s}$ and $0 \mathrm{~s}$ in two independently scrollable image memories. Set the look-up tables to compute XOR of the pixels of the two images; that is, the table should be set to 0 ("off") if and only if both images send the same value and to 255 ("on") otherwise. Then a new, equally independent, random stimulus will appear for each scroll position of the second image. Thus, $512 \times 512$ random images can be created, enough for $2 \mathrm{~h}$ of novel noise stimuli at 30 frames per second. The sequence of scroll positions should be random also. If it is sequential, random regularities tend to become apparent because they are partially preserved across a few frames.

I close this section by illustrating some trade-offs that sometimes arise in an actual case. Recall the study mentioned above that involved simulating dots on a rotating sphere via the 40 -frame-resolution animation technique. In order to show the individual bit-planes for each frame, an entire 256-element look-up table needed to be written. More data had to be passed to select the particular image memory and where, in space, to zoom around. Although the total amount of information required for this was only 297 16-bit words and I was accustomed to an older machine (the PDP-11/60) that could easily transmit that many words in the $2-3$ msec it had available during the time the screen was blanked, the newer VAX11/780 could not handle it. The VAX would transmit as much as it could during one blank period, then a rather strange image would be created, and then it would transmit the rest during the next blank period.

The solution is simple and surprisingly effective. I no longer use all eight bit-planes per image; I now use seven. This reduction results in a dramatic reduction in the amount of information that needs to be communicated for each frame-almost by half. Yet the number of discrete frames available is only reduced by $13 \%$. The reason is that, instead of having to write 256 elements in the table, only 128 elements are required for the seven planes. The total information required dropped from 297 to 169 words, and the procedure ran smoothly, in both senses of the word.

The lesson to be gleaned from the examples in Case 5 is that, although digital image processors were not created to generate moving images, combining imagination with some sacrifice of spatial, intensity, temporal, and/or structural resolution may make moving images possible.

\section{FROM FANTASY TO REALITY}

General-purpose digital image-processing systems are becoming a standard and virtually essential piece of laboratory equipment for vision research. The general discussion and case studies presented here should provide the reader with some feel for the technical limitations and uses of such equipment. There will continue to be improvements, perhaps even at the behest of vision workers. But the completely general psychophysical device for studying vision will not be with us in the near future. Studying achromatic spatial contrast one day is followed by interest in color constancy, reaction time, or cortical evoked potentials the next. Concern with abstract, mathematically elegant, and theoretically important stimuli, such as Gabor functions, may be replaced by interest in object recognition and motion processing of cinematic passages from $E T$. In a more practical example, the goal of visually communicating American Sign Language over current telephone bandwidths will require new hardware to im- 
plement novel approaches to image compression (Daugman, 1985; Sperling, 1980). I hope that the occasional fantasy of psychophysical omnipotence does not lull the researcher away from the desire to explore novel approaches to image generation.

\section{REFERENCES}

AdeLsON, E. H., \& BERGEN, J. R. (1985). Spatiotemporal energy models for the perception of motion. Journal of the Optical Society of America, A, 2, 284-299.

Anstis, S., \& Cavanagh, P. (1983). A minimum motion technique for judging equiluminance. In J. D. Mollon \& L. T. Sharpe (Eds.), Colour vision physiology and psychophysics (pp. 155-166). London: Academic Press.

Cavanagh, P., Maurer, D., Lewis, T., Macleod, D. A. I., \& MATHER, G. (1986). Computer-generated screening test for colorblindness. Color Research \& Applications, 11, S63-S66.

Citron, M. C., \& Emerson, R. C. (1983). White noise analysis of cortical directional selectivity in cat. Brain Research, 279, 271-277.

CowaN, W. B. (1983). Discreteness artifacts in raster display systems. In J. D. Mollon \& L. T. Sharpe (Eds.), Colour vision physiology and psychophysics (pp. 145-153). London: Academic Press.

DAugman, J. G. (1985). Image analysis by local 2-D spectral signatures. Joumal of the Optical Society of America, A, 2, 74.

GREEN, D. M. (1976). An introduction to hearing. Hillsdale, NJ: Erlbaum.

HABER, R. N. (1986). Flight simulation. Scientific American, 255(1), 96-103.
Kelly, D. H., \& SAvoie, R. E. (1973). A study of sine-wave contrast sensitivity by two psychophysical methods. Perception \& Psychophysics, 14, 313-318.

LAND, E. H. (1974). The retinex theory of colour vision. Proceedings of the Royal Institution of Great Britain, 47, 23-58.

MACLeod, D. A. I. (1986). Computer controlled color displays in vision research: Possibilities and problems. Color Research \& Applications, 11, S45-S46.

McCann, J. J., McKee, S. P., \& TAYlor, T. H. (1976). Quantitative studies in retinex theory. Vision Research, 16, 445-448.

MCKEE, S. P., \& WELCH, L. (1985). Spatial recruitment in the discrimination of velocity. Journal of the Optical Society of America, A, 2, 243-251.

Mulligan, J. B. (1986). Minimizing quantization errors in digitallycontrolled CRT displays. Color Research \& Applications, 11, S47-S51.

Pinker, S. (1984). Visual cognition: An introduction. Cognition, 18, 1-63.

Robertson, B. (1986). Pixar goes commercial in a new market. Computer Graphics World, 9(6), 61-70.

RoDIrCK, R. W. (1983). Raster-based colour stimulators. In J. D. Mollon \& L. T. Sharpe (Eds.), Colour vision physiology and psychophysics (pp. 131-144). London: Academic Press.

SAvoY, R. L. (1980). Visual studies with low frequency gratings (Unpublished doctoral dissertation) Harvard University, Cambridge, MA.

Savoy, R. L. (1984). "Extinction" of the McCollough effect does not transfer interocularly. Perception \& Psychophysics, 36, 571-576.

SavoY, R. L., \& MCCANN, J. J. (1975). Visibility of low-spatialfrequency sine-wave targets: Dependence on number of cycles. Joumal of the Optical Society of America, 65, 343-350.

SPERLING, G. (1980). Bandwidth requirements for video transmission of American Sign Language and finger spelling. Science, 210, 797-799. 